\title{
Review Article \\ The Effect of Teriparatide on Fracture Healing of Osteoporotic Patients: A Meta-Analysis of Randomized Controlled Trials
}

\author{
Shenghan Lou, Houchen Lv, Guoqi Wang, Licheng Zhang, Ming Li, \\ Zhirui Li, Lihai Zhang, and Peifu Tang \\ Department of Orthopedics, General Hospital of Chinese PLA, Beijing, China \\ Correspondence should be addressed to Peifu Tang; pftang301@163.com
}

Received 17 January 2016; Accepted 30 May 2016

Academic Editor: Hai-Feng Pan

Copyright (c) 2016 Shenghan Lou et al. This is an open access article distributed under the Creative Commons Attribution License, which permits unrestricted use, distribution, and reproduction in any medium, provided the original work is properly cited.

\begin{abstract}
Purpose. This meta-analysis is to assess the effectiveness of teriparatide in fracture healing and clinical function improvement of the osteoporotic patients. Methods. We searched PubMed, Embase, Web of Science, and the Cochrane databases for randomized and quasi-randomized controlled trials comparing teriparatide to placebo, no treatment, or comparator interventions in the osteoporotic patients. Results. Five studies with 251 patients were included. Patients treated with teriparatide therapy had a significant shorter radiological fracture healing time compared with those in the control group (mean difference [MD] -4.54 days, $95 \%$ confidence interval $[\mathrm{CI}]-8.80$ to -0.28 ). Stratified analysis showed that lower limb group had significant shorter healing time (MD -6.24 days, 95\% CI -7.20 to -5.29 ), but upper limb group did not (MD -1 days, 95\% CI -2.02 to 0.2 ). Patients treated with teriparatide therapy showed better functional outcome than those in the control group (standardized mean difference [SMD] $-1.02,95 \%$ CI -1.81 to -0.22 ). Patients with therapy duration over 4 weeks would have better functional outcome (SMD -1.68 , 95\% CI -2.07 to -1.29$)$. Conclusions. Teriparatide is effective in accelerating fracture healing and improving functional outcome of osteoporotic women. However, more clinical studies are warranted in order to determine whether the results are applicable to males and the clinical indications for teriparatide after osteoporotic fractures.
\end{abstract}

\section{Introduction}

Bisphosphonates, the synthetic analogues of pyrophosphate [1], are the most widely used medications for the treatment of osteoporosis $[2,3]$. The mechanism of bisphosphonates is that bisphosphonates accumulate in bone by binding to mineral crystals and bisphosphonates like alendronate preferentially deposit, not in newly formed bone, but beneath osteoclasts. The key pharmacological action of bisphosphonates is the inhibition of osteoclast-mediated bone resorption. Bisphosphonates inhibit the formation and aggregation of calcium phosphate crystals to prevent bone loss and improve bone strength [4]. However, a long-term use of bisphosphonates may decrease bone formation, which is attributed to the long-term inhibition of osteoclasts [5]. Osteoclasts play an important role in remodeling of the callus into cortical bone. Thus, bisphosphonates may produce adverse effects on the healing process of fractures $[6,7]$.
Parathyroid hormone (PTH), an 84-amino acid peptide secreted by the parathyroid gland, is an important systemic regulator of calcium homeostasis [8]. It has been demonstrated that intermittent administration of PTH leads to an anabolic effect on bone [9]. Teriparatide, a synthetic polypeptide hormone consisting of the 1-34 fragment of human parathyroid hormone, retains most of the biological activities of PTH [10]. Intermittent administration of teriparatide also has the anabolic effects that stimulate bone formation and activate bone remodeling [11], improving the microarchitecture of trabecular bone and cortical bone [12]. Previous studies reported that teriparatide also increased bone mineral density and decreased risk of vertebral and nonvertebral fractures [13-16]. Meanwhile, teriparatide was the only anabolic drug for osteoporosis adopted by the Food and Drug Administration [17].

Previous studies reported favourable changes in bone mineral content, structure, and microarchitecture after 
teriparatide treatment due to the anabolic effect of this drug $[18,19]$. As intermittent administration of teriparatide can stimulate bone formation, it seems reasonable to assume that teriparatide might accelerate fracture healing simultaneously in osteoporosis cases with fractures [20-23]. Recently, some studies about osteoporotic fracture cases indicated a beneficial effect of teriparatide [24-26], but the latest two randomized controlled trials (RCTs) showed that there were no statistically significant differences between the group treated with teriparatide and the group treated with placebo $[27,28]$. As evidence-based evaluation of this issue is limited, the effect of teriparatide on osteoporotic fracture healing remains controversial [29].

Therefore, to determine whether teriparatide accelerates fracture healing in the osteoporotic patients, we performed a meta-analysis of RCTs. In this study, we evaluated the effectiveness of teriparatide in osteoporotic fracture healing and clinical function improvement. The results of this study would elucidate whether teriparatide would be effective in inducing fracture healing and improving functional outcome for the osteoporotic fracture patients.

\section{Material and Methods}

2.1. Search Strategy. A search of PubMed, the Cochrane Library, and Embase was performed in November 2015 for studies published between 1966 and October 2015, using the following combination of terms: "teriparatide" or "Parathyroid Hormone" or "forteo" or "PTH (1-34)" or "PTH (1-84)" or "parathormone" or "parathyrin" and "fractures healing" or "healing" and "fractures, bone" or "broken bone" or "bone fracture" or "fractures." Google Scholar was also used to screen relevant literature, and the reference list was manually searched from all the relevant original research and review articles to identify additional potentially eligible studies. There were no language restrictions on trial eligibility.

2.2. Selection Criteria. Studies were included if they met the following criteria: (1) study design was a RCT; (2) participants had osteoporosis with fractures, and (3) the intervention was teriparatide initiation compared with placebo, no treatment control group, or comparator interventions, such as vitamin $\mathrm{D}$, bisphosphonates, analgesics, and calcium.

Studies were excluded if they met the following criteria: (1) participants younger than 50 years of age, (2) contraindication to any of the study drugs, formerly or currently on any of them, (3) serum calcium above the reference level and liver enzymes more than double of the upper reference level, (4) history of tumor or chemotherapy, bone metastases, open or pathologic fractures, known metabolic bone disease, rheumatoid arthritis or chronic renal failure, joint disease, or any disease affecting bone metabolism; (5) the articles which were not available or had repeated data.

2.3. Data Collection and Endpoints. The appropriate articles were verified by two independent investigators (S. Lou and G. Wang). In case of disagreement between the two investigators, a third one was consulted. We extracted information of the participants' characteristics, type of fracture and treatment, time of teriparatide initiation and treatment, follow-up, time of radiological fracture healing, and functional outcome from each study.

The primary endpoint was the time of fracture healing, as determined by radiography, which was defined as the time of cortical bridging in three of four cortices. The functional outcome was defined as an improvement in mobility at week 12 and assessed with the Timed "Up and Go" (TUG) test or the self-administered "Patient-Rated Wrist Evaluation" (PRWE) questionnaire or "disabilities of the arm, shoulder, and hand" (DASH) score or the "Johanson Hip Rating Questionnaire” (JHRQ) [27, 28, 30, 31].

2.4. Quality Assessment. The RCTs were evaluated by the "Cochrane Collaboration's tool for assessing the risk of bias," which included the following aspects: (1) randomsequence generation (selection bias); (2) allocation concealment (selection bias); (3) blinding of participants and personnel (performance bias); (4) blinding of outcome assessment (detection bias); (5) incomplete outcome data (attrition bias); (6) selective reporting (reporting bias); (7) other bias.

2.5. Grading Quality of Evidence. Two authors (SHL, HCL) independently evaluated the quality of evidence for primary and secondary outcomes according to the Grading of Recommendations Assessment, Development, and Evaluation (GRADE) [32] methodology for risk of bias, inconsistency, indirectness, imprecision, and publication bias. The assessment results were classified as very low, low, moderate, or high. Summary tables were constructed using the GRADE Profiler (version 3.6).

2.6. Statistical Analysis. Statistical analyses were performed with Review Manager Software (version 5.3; the Nordic Cochrane Center, the Cochrane Collaboration, Copenhagen, Denmark). Continuous outcomes were expressed as mean difference (MD) and 95\% CI. Dichotomous outcomes were expressed as OR and 95\% CI. To assess heterogeneity in results of individual studies, we used Cochran's $Q$ statistic, $I^{2}$ statistic $\left(I^{2}>50 \%\right.$ was used as a threshold indicating significant heterogeneity), and $P$ values $(P$ value $<0.10$ was used as a threshold indicating significant heterogeneity) [33]. A fixed effects model was applied in the meta-analysis. However, a random effects model was used when significant heterogeneity was found [34]. In the planning stage, sensitivity analysis would be performed by omission of each study to evaluate stability of the results if heterogeneous studies existed. Funnel plots were used to assess for publication bias. All tests were two-tailed and $P$ value $<0.05$ was deemed statistically significant.

\section{Results}

3.1. Literature Search and Characteristics. Our search strategy identified 296 relevant articles, the titles and abstracts of which were screened for inclusion. The full text of 7 articles was retrieved, 5 of which $[27,28,30,31,35]$ met the inclusion criteria. A manual search of the reference list within these 
TABLE 1: Characteristics of included studies.

\begin{tabular}{|c|c|c|c|c|c|c|}
\hline \multirow{2}{*}{ Studies } & \multirow{2}{*}{ Number of patients } & \multicolumn{2}{|c|}{ Age/years } & \multirow{2}{*}{ Type of fracture } & \multicolumn{2}{|c|}{ Sex } \\
\hline & & Mean & $\mathrm{SD}$ & & $\mathrm{F}$ & $\mathrm{M}$ \\
\hline Kanakaris et al. 2015 [27] & 30 & 75 & 8.89 & Hip fractures (low energy) & 24 & 6 \\
\hline Johansson 2016 [28] & 40 & 68 & 8.6 & Proximal humeral fracture & 40 & 0 \\
\hline Song et al. 2012 [35] & 14 & 76.2 & 8.2 & Femoral comminuted fracture & 12 & 2 \\
\hline Peichl et al. 2011 [30] & 65 & 82.3 & 4.1 & Pelvic fracture & 65 & 0 \\
\hline Aspenberg et al. 2010 [31] & 102 & 61.4 & 8.6 & Distal radius fracture & 102 & 0 \\
\hline
\end{tabular}

F, female; $\mathrm{M}$, male.

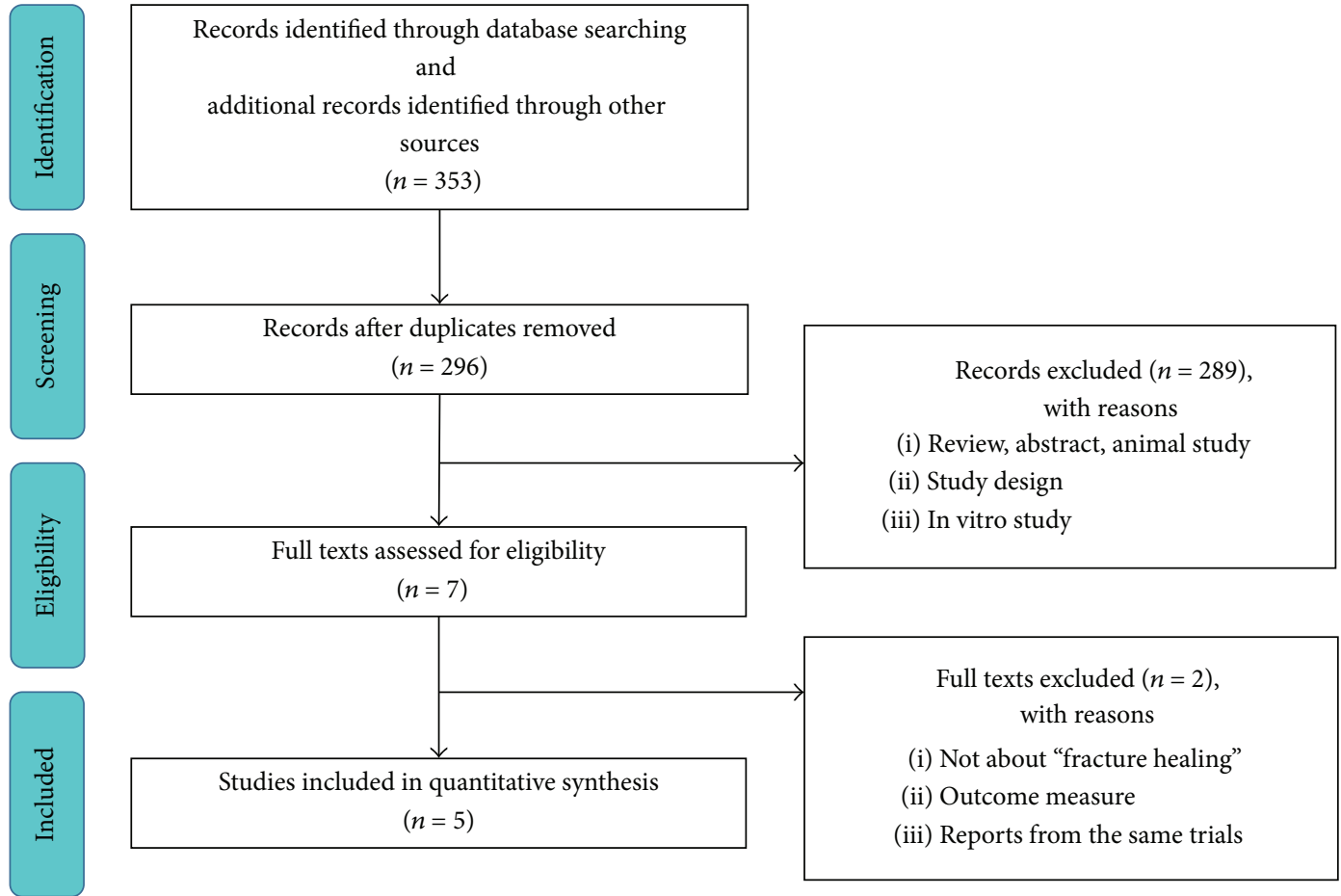

FIgURE 1: Flow diagram shows the process of literature selection.

studies did not yield any additional eligible studies. Figure 1 illustrates the process of study selection.

A total of 251 patients were randomly assigned in the 5 trials included in this meta-analysis. There were 125 patients in the experimental group, and 126 in the control group. Regarding sex, 3.1\% $(n=8)$ of the patients were male and $96.9 \%(n=243)$ were female. The overall mean age was 70.3 years. As for the fracture type, two trials had upper limb fractures, including distal radial fractures and proximal humeral fractures, and three trials had lower limb fractures, including pelvic fractures and hip fractures. The detailed characteristics of the included studies are listed in Table 1.

The experimental group included teriparatide or PTH184. There were differences in pharmacokinetics and actions between these two kinds of PTH, which resulted in the anabolic effect of $100 \mu \mathrm{g}$ PTH1-84 being equal to $20 \mu \mathrm{g}$ teriparatide. The control group included placebo, no treatment, or other drugs interventions. The study of Kanakaris et al. [27] had two kinds of control groups, either only vitamin D and calcium or those plus bisphosphonate (Alendronate, $70 \mathrm{mg}$ orally). Another study [31] had two kinds of experimental groups, among which $20 \mu \mathrm{g}$ or $40 \mu \mathrm{g}$ teriparatide was taken. Both of them were united as one in these two studies. The treatment time of teriparatide varied from 4 weeks to 24 months in the experimental group. The detailed characteristics are listed in Table 2.

3.2. Methodological Quality. The methodological quality of the RCTs is presented in Figure 2. The rate of patients lost to follow-up was appraised, and the dropout rate in only one trial was high (60\%) [27].

3.3. Fracture Healing Time. The time of radiological fracture healing was defined as the time of cortical bridging in three of four cortices. Three trials met the inclusion criteria of this meta-analysis [30, 31, 35]. According to the results, patients who were treated with teriparatide had statistically significant difference in radiological fracture healing time compared 
TABLE 2: Detail of intervention.

\begin{tabular}{|c|c|c|c|c|c|c|}
\hline \multirow{2}{*}{ Studies } & \multicolumn{2}{|c|}{ Intervention } & \multirow{2}{*}{$N_{e}$} & \multirow{2}{*}{$N_{c}$} & \multirow{2}{*}{ Treatment time } & \multirow{2}{*}{$\begin{array}{l}\text { Time of } \\
\text { initiation }\end{array}$} \\
\hline & EG & CG & & & & \\
\hline \multirow{2}{*}{ Kanakaris et al. 2015 [27] } & \multirow{2}{*}{ Teriparatide $20 \mu \mathrm{g}$} & Alendronate & \multirow{2}{*}{9} & \multirow{2}{*}{21} & \multirow{2}{*}{4 weeks } & \multirow{2}{*}{-} \\
\hline & & $70 \mathrm{mg}$ or vitamin $\mathrm{D}$ and calcium & & & & \\
\hline \multirow{2}{*}{ Johansson 2016 [28] } & Teriparatide $20 \mu \mathrm{g}$; analgesics & Analgesics & \multirow{2}{*}{20} & \multirow{2}{*}{20} & \multirow{2}{*}{4 weeks } & \multirow{2}{*}{$<10$ days } \\
\hline & Physiotherapy & Physiotherapy & & & & \\
\hline Song et al. 2012 [35] & Teriparatide $20 \mu \mathrm{g}$ & No therapy & 7 & 7 & 3 months & - \\
\hline \multirow{2}{*}{ Peichl et al. 2011 [30] } & PTH1-84 $100 \mu \mathrm{g}$; calcium $1000 \mathrm{mg}$ & Calcium $1000 \mathrm{mg}$ & \multirow{2}{*}{21} & \multirow{2}{*}{44} & \multirow{2}{*}{24 months } & \multirow{2}{*}{$<2$ days } \\
\hline & Vitamin D $800 \mathrm{IU}$ & Vitamin D $800 \mathrm{IU}$ & & & & \\
\hline \multirow[t]{2}{*}{ Aspenberg et al. 2010 [31] } & Teriparatide & \multirow[t]{2}{*}{ Placebo } & \multirow[t]{2}{*}{68} & \multirow[t]{2}{*}{34} & \multirow[t]{2}{*}{8 weeks } & \multirow{2}{*}{$<10$ days } \\
\hline & 20 or $40 \mu \mathrm{g}$ & & & & & \\
\hline
\end{tabular}

$N_{e}$ : number in experimental group, $N_{c}$ : number in control group.

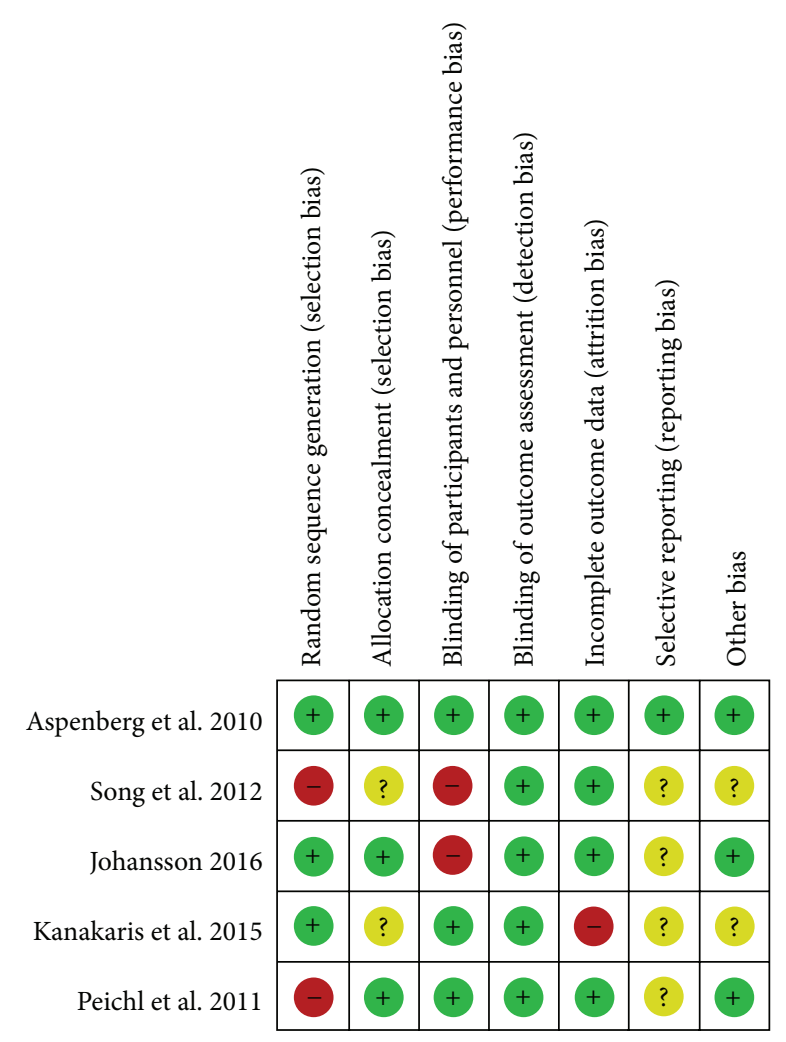

FIgURE 2: The methodological quality of the RCTs. Risk of bias summary. "+" means low risk; "?" means unclear risk; "_" means high risk.

with the control group ( $\mathrm{MD}-4.54,95 \% \mathrm{CI}-8.80$ to $-0.28 ; I^{2}$ of heterogeneity 96\%, $P<0.00001$; random effects model) (Figure 3). As $I^{2}=96 \%$, apparently over 50\%, indicated significant heterogeneity, we further performed a sensitivity analysis and found that one trial [31] significantly affected the pooled MD. Therefore, a subgroup analysis, consisting of upper limb group (MD $-1,95 \% \mathrm{CI}-2.02$ to $0.2 ; P=0.05$; random effects model) and lower limb group (MD -6.24, 95\% CI -7.20 to $-5.29 ; I^{2}$ of heterogeneity $0 \%, P=0.70$; random effects model), was performed (Figure 3). A visible difference was found between the upper limb and the lower limb.

3.4. Functional Outcome. The functional outcome was defined as an improvement in mobility at week 12 and assessed with the TUG test [30] or the self-administered PRWE questionnaire [31] or DASH score [28] or the JHRQ [27]. Because of the different measurement methods, a standardized mean difference method was used. Four trials were eligible for the meta-analysis of the functional outcome $[27,28,30,31]$. Patients who were treated with teriparatide showed significantly better functional outcome than those in the control group (SMD $-1.02,95 \% \mathrm{CI}-1.81$ to $-0.22 ; I^{2}$ of heterogeneity $85 \%, P=0.00002$; random effects model) (Figure 4 ). In view of $I^{2}=85 \%$ symbolized significant heterogeneity, we performed a subgroup analysis, in which one group represented that treatment time exceeded 4 weeks (SMD $-1.68,95 \%$ CI -2.07 to $-1.29 ; I^{2}$ of heterogeneity $0 \%$, $P=0.55$; random effects model) and the other represented that treatment time was equal to 4 weeks (SMD $-0.31,95 \%$ CI -0.81 to $0.18 ; I^{2}$ of heterogeneity $0 \%, P=0.34$; random effects model) (Figure 4). The duration of treatment was the key factor for the function outcome.

3.5. Sensitivity Analysis. Sensitivity analysis was performed by omission of each study to evaluate stability of the results if heterogeneous studies existed. The sensitivity analysis for the fracture healing time showed that the study of Aspenberg et al. [31] significantly affected the pooled MD (Table 3). The sensitivity analysis for functional outcome showed that any study did not significantly affect the pooled MD (Table 4).

3.6. Publication Bias and GRADE Profile Evidence. For the meta-analysis of fracture healing and functional outcome, there was no evidence showing obvious publication bias by examining the symmetry of the funnel plot (Figures 5 and 6).

GRADE evidence profiles for the primary and secondary outcomes were shown in Table 5. The most common reasons for the decreased level of evidence were the heterogeneity and suspected publication bias. 
TABLE 3: Sensitivity analyses based on various exclusion criteria for fracture healing time.

\begin{tabular}{lcccccccc}
\hline Excluded trial & Number of trials & Number of patients & EG & CG & MD (95\% CI) & $P$ value for MD & $I^{2}, \%$ & $\begin{array}{c}P \text { value for } \\
\text { heterogeneity }\end{array}$ \\
\hline Aspenberg et al. 2010 [31] & $2[30,35]$ & 79 & 28 & 51 & $-6.24[-7.20,-5.29]$ & $<0.000001$ & 0 & 0.7 \\
Song et al. 2012 [35] & $2[30,31]$ & 147 & 76 & 71 & $-3.60[-8.70,1.49]$ & 0.17 & 98 & $<0.000001$ \\
Peichl et al. 2011 [30] & $2[31,35]$ & 96 & 62 & 34 & $-3.66[-9.59,2.27]$ & 0.23 & 86 & 0.008 \\
\hline
\end{tabular}

EG, experimental group; CG, control group.

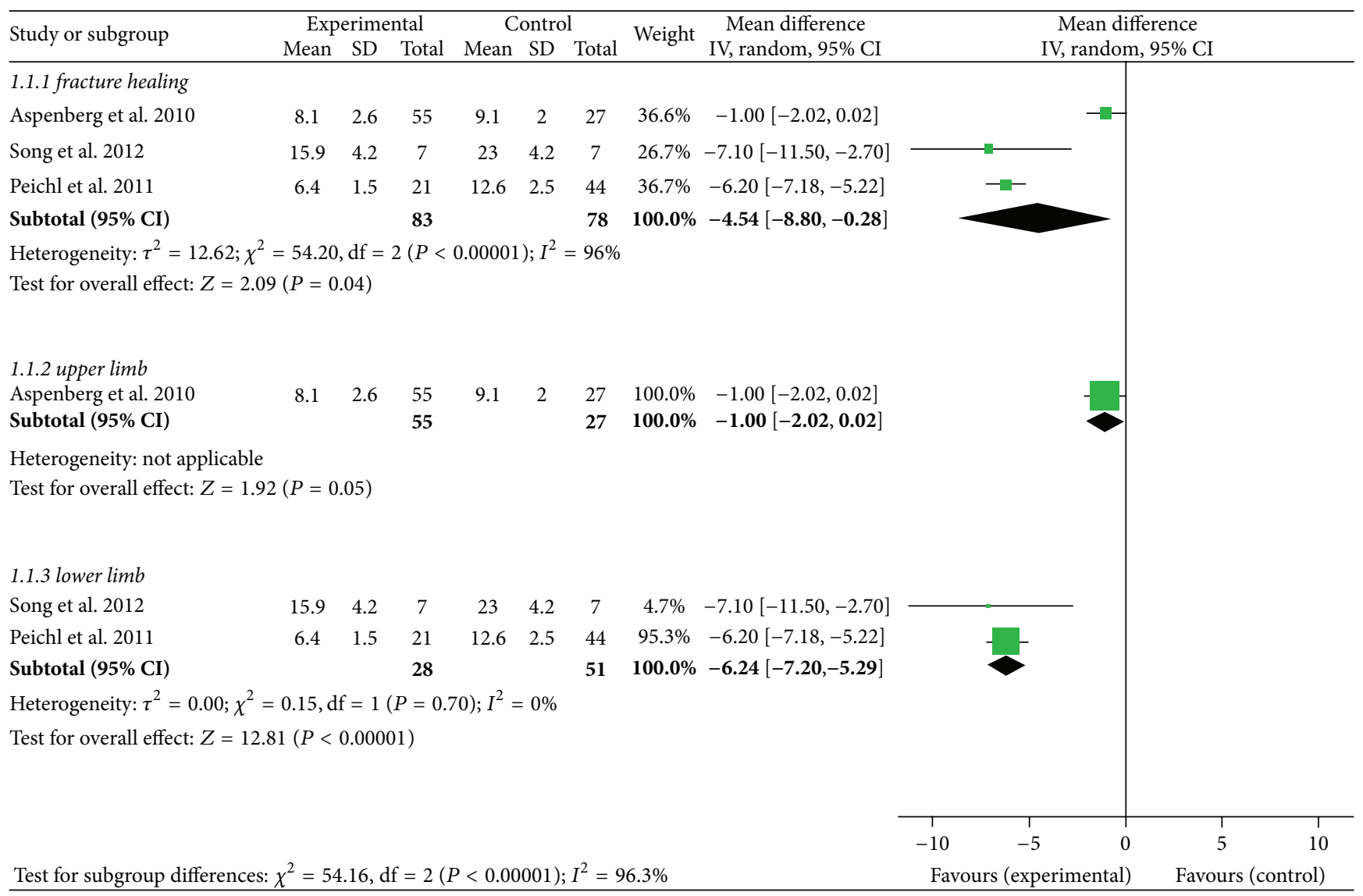

FIGURE 3: Forest plot for radiological fracture healing time.

\section{Discussion}

4.1. Main Findings. As far as we know, this is the first metaanalysis to examine the effect of teriparatide on fracture healing and functional outcome of the osteoporotic patients. Our meta-analysis comprehensively and systematically reviewed the current available literature in regard to the teriparatide therapy for osteoporotic patients and found that (1) teriparatide therapy promoted osteoporotic fracture healing and the evidence of outcomes was confirmed by the GRADE system, although this evidence came from only three trials; (2) teriparatide therapy improved function outcome, which was confirmed by the GRADE system as well.

Furthermore, subgroup analysis showed that the effect of teriparatide was significantly related to the time of drug application and the site of fractures. Fractures healed by different mechanisms, depending on the location of fractures. One possible explanation is that the anabolic effect of teriparatide is further enhanced when bone is subjected to mechanical stimulation, and fractures of the load-bearing bone might be more susceptible to teriparatide. Similarly, compared with 4 -week treatment $[27,28]$ or 8 -week treatment [31], 24-month treatment [30] produced statistically significant results in terms of fracture healing and functional improvement. Although teriparatide could improve early callus formation [36], better effects may be seen with a longer duration of treatment.

4.1.1. Implications for Clinical Practice. Fracture healing is a very complex process that involves both resorptive and formative processes. For osteoporotic patients, the long 
TABLE 4: Sensitivity analyses based on various exclusion criteria for functional outcome.

\begin{tabular}{lcccccccc}
\hline Excluded trial & Number of trials & Number of patients & EG & CG & MD (95\% CI) & $P$ value for MD & $\begin{array}{c}I^{2}, \% \\
\text { heterogeneity }\end{array}$ \\
\hline Aspenberg et al. 2010 [31] & $3[27,28,30]$ & 134 & 49 & 85 & $-0.80[-1.87,0.27]$ & 0.14 & 87 & 0.0005 \\
Johansson 2016 [28] & $3[27,30,31]$ & 186 & 91 & 95 & $-1.18[-2.14,-0.22]$ & 0.02 & 86 & 0.0008 \\
Kanakaris et al. 2015 [27] & $3[28,30,31]$ & 195 & 101 & 94 & $-1.32[-2.06,-0.58]$ & 0.0005 & 80 & 0.008 \\
Peichl et al. 2011 [30] & $3[27,28,31]$ & 160 & 89 & 71 & $-0.74[-1.69,0.21]$ & 0.13 & 85 & 0.001 \\
\hline
\end{tabular}

EG, experimental group; CG, control group.

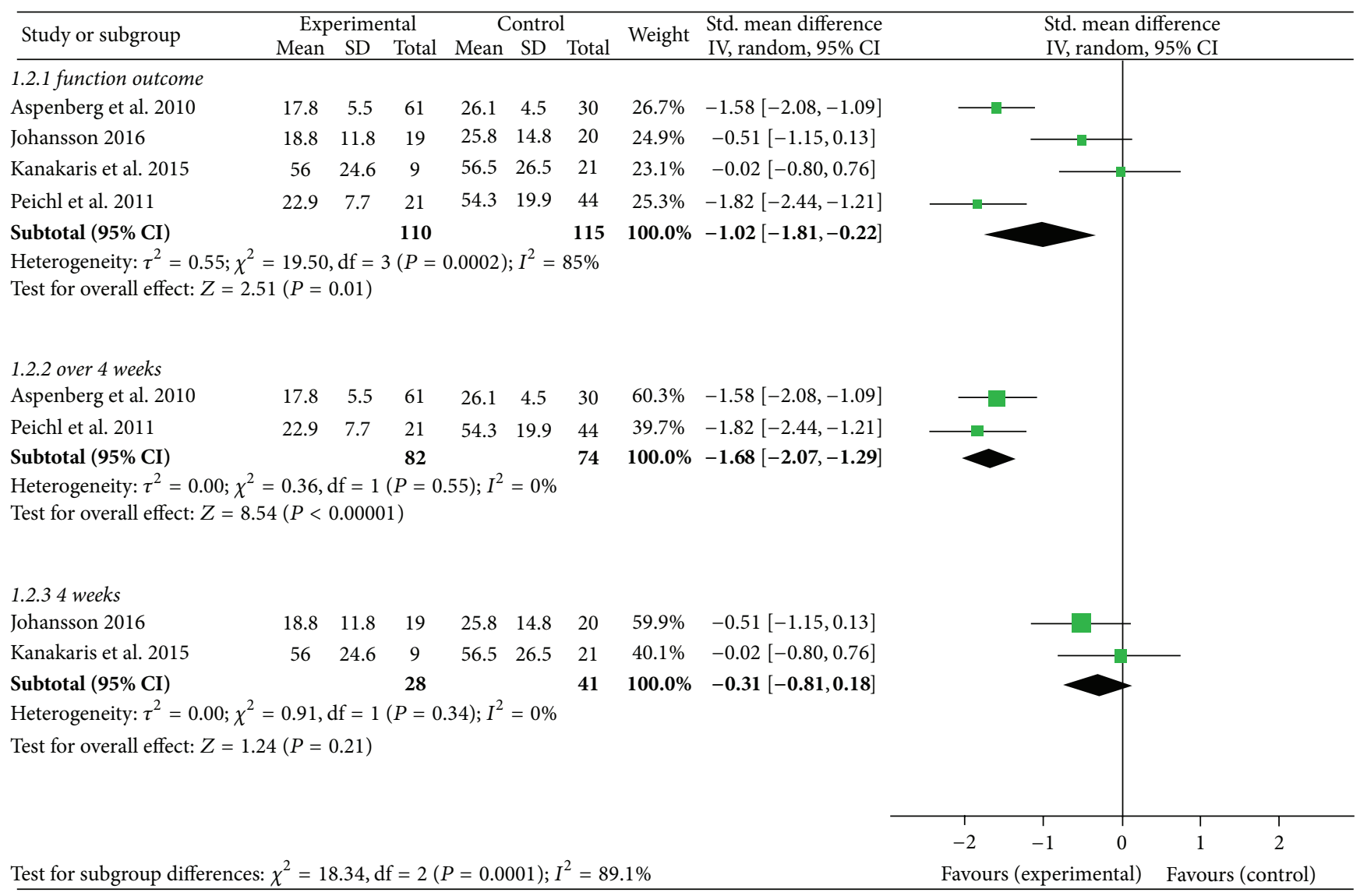

FIGURE 4: Forest plot for functional outcome.

bone is ductile and shows plastic deformation before fractures. The main effect of teriparatide is to stimulate bone formation without stimulating bone resorption, which is called "anabolic window." There are multiple mechanisms for teriparatide to promote fracture healing, including promoting proliferation and differentiation of mesenchymal stem cell, chondroprogenitors and osteoprogenitors, chondrocyte maturation, production of bone matrix proteins, and osteoclastogenesis. During fracture healing, it can increase callus formation by increasing the induction of proliferation and differentiation of osteoprogenitors and chondroprogenitors [37, 38]. It also enhances callus formation and callus remodeling by stimulating bone matrix protein synthesis and osteoclastogenesis [38]. In addition, teriparatide increases fracture callus size and expression of types II and X collagen via the Wnt/ $\beta$-catenin signaling pathway [39].

The primary outcomes of our meta-analysis showed that, in case of osteoporotic fractures, teriparatide is a viable therapy that is not only able to treat the underlying osteoporosis but also able to accelerate fracture healing, especially for osteoporotic women. Correspondingly, it has been discovered that there are different effects between upper limbs and lower limbs via a subgroup analysis in our metaanalysis, and the lower limb group has a better result than the upper limb group.

To date, no systemic treatment is approved for fracture healing. Impaired healing of fractures delays the rehabilitation process, which influences life quality of the patients. At 


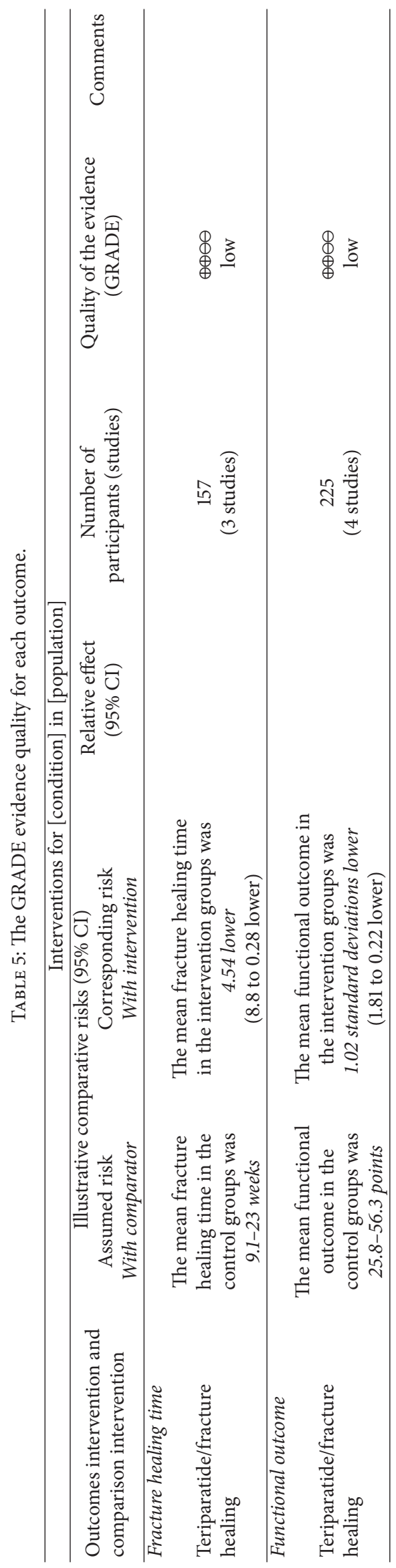




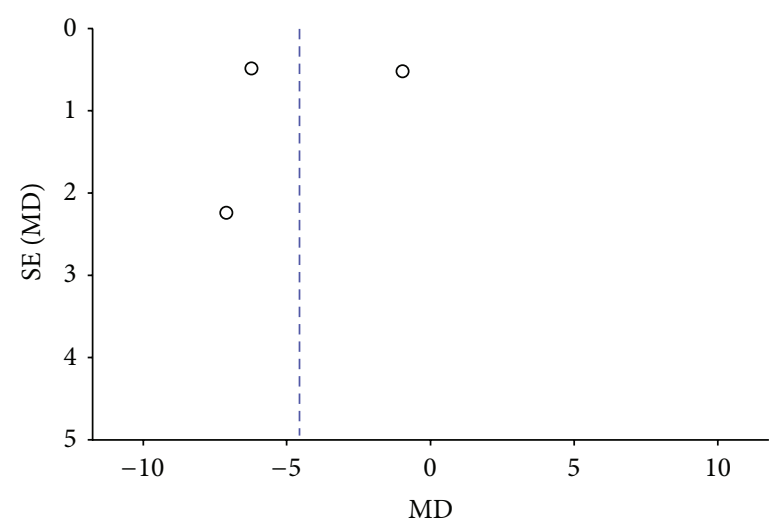

Subgroups

$\circ$ Fracture healing

FIGURE 5: Funnel plot for fracture healing.

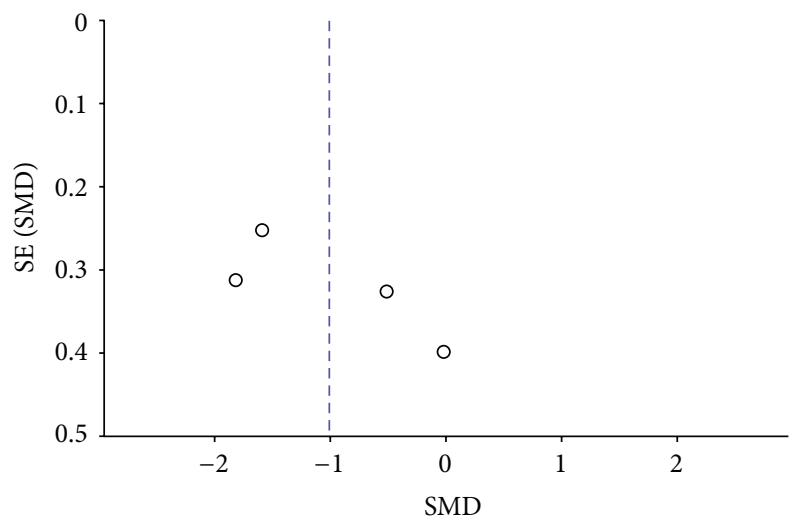

Subgroups

○ Function outcome

FIGURE 6: Funnel plot for functional outcome.

the same time, the associated costs cause an economic burden to both the society and the patients. Teriparatide therapy accelerates healing, which allows patients to return to normal life and work faster, and it reduces the medical consumption and chronic morbidity associated with long-term treatment. Furthermore, it can be applied to any types of fractures, including those that will be treated nonsurgically, can be commenced at any time, and can be applied through the entire healing period as well. As teriparatide therapy can promote osteoporotic fracture healing and improve function outcome, we suspect that teriparatide may prove to be useful in the stimulation of implant anchoring and fixation for both dental and orthopedic implants. Likewise, it may prove to be useful in fractures which have a high risk of delayed union or nonunion, as well as a very high degree of associated disability. Some studies have been started to explore related issues [40-45], but studies are still limited, and most of them are case reports. The hypotheses still need evaluation by high-quality randomized controlled trials.
4.1.2. Strengths and Limitations. A major strength of this meta-analysis was compliance with the PRISMA guidelines and the recommendations of the Cochrane Collaboration, although our study was not registered with protocol. Additionally, the GRADE system was used to evaluate the quality of evidence for the outcomes in this meta-analysis.

There were, however, several limitations of this metaanalysis. First, only five articles were included, and sample size of the most included studies was small. Second, two studies $[27,31]$ contained multiple groups. We combined these groups together because the effect of teriparatide was studied in a broad sense, not compared with a particular medication. Third, publication bias was unclear due to the limited studies. Additionally, as more than $75 \%$ of osteoporotic fractures occurred in women [46] and only six patients of the study were males, the primary result seemed more applicable to osteoporotic women. Some more RCTs are needed to determine whether the results are applicable to males. Finally, in spite of adopting the proven methods [47] to estimate the missing data, a more detailed and comprehensive analysis was restricted.

\section{Conclusion}

There is no reason to believe that teriparatide will not be effective in improving functional outcome and inducing fracture healing in the osteoporotic women. However, researches in this field are not enough, some more clinical studies are needed to increase the quality of evidence and determine whether the results are applicable to males. Meanwhile, a number of clinical studies are warranted in order to determine the usefulness of teriparatide and the clinical indications for the use of teriparatide in the treatment of the osteoporotic fracture healing.

\section{Disclosure}

The current address of Shenghan Lou is Department of Orthopedics, Chinese PLA General Hospital, No. 28 Fuxing Road, Beijing 100853, China.

\section{Competing Interests}

The authors declare that they have no competing interests.

\section{Authors' Contributions}

Shenghan Lou and Houchen Lv contributed equally to this work. Guoqi Wang and Licheng Zhang also contributed equally to this work.

\section{References}

[1] G. A. Rodan and H. A. Fleisch, "Bisphosphonates: mechanisms of action," Journal of Clinical Investigation, vol. 97, no. 12, pp. 2692-2696, 1996.

[2] Y. A. Weil, G. Rivkin, O. Safran, M. Liebergall, and A. J. Foldes, "The outcome of surgically treated femur fractures associated 
with long-term bisphosphonate use," Journal of TraumaInjury, Infection and Critical Care, vol. 71, no. 1, pp. 186-190, 2011.

[3] C. V. Odvina, S. Levy, S. Rao, J. E. Zerwekh, and D. S. Rao, "Unusual mid-shaft fractures during long-term bisphosphonate therapy," Clinical Endocrinology, vol. 72, no. 2, pp. 161-168, 2010.

[4] F. Von Knoch, C. Jaquiery, M. Kowalsky et al., "Effects of bisphosphonates on proliferation and osteoblast differentiation of human bone marrow stromal cells," Biomaterials, vol. 26, no. 34, pp. 6941-6949, 2005.

[5] S. A. Jamal, N. Dion, and L.-G. Ste-Marie, "Atypical femoral fractures and bone turnover," The New England Journal of Medicine, vol. 365, no. 13, pp. 1261-1262, 2011.

[6] C. V. Odvina, J. E. Zerwekh, D. S. Rao, N. Maalouf, F. A. Gottschalk, and C. Y. C. Pak, "Severely suppressed bone turnover: a potential complication of alendronate therapy," Journal of Clinical Endocrinology and Metabolism, vol. 90, no. 3, pp. 1294-1301, 2005.

[7] Y. Cao, S. Mori, T. Mashiba et al., "Raloxifene, estrogen, and alendronate affect the processes of fracture repair differently in ovariectomized rats," Journal of Bone and Mineral Research, vol. 17, no. 12, pp. 2237-2246, 2002.

[8] S.-I. Harada and G. A. Rodan, "Control of osteoblast function and regulation of bone mass," Nature, vol. 423, no. 6937, pp. 349355, 2003.

[9] R. L. Jilka, "Molecular and cellular mechanisms of the anabolic effect of intermittent PTH," Bone, vol. 40, no. 6, pp. 1434-1446, 2007.

[10] M. Kneissel, A. Boyde, and J. A. Gasser, "Bone tissue and its mineralization in aged estrogen-depleted rats after longterm intermittent treatment with parathyroid hormone $(\mathrm{PTH})$ analog SDZ PTS 893 or human PTH(1-34)," Bone, vol. 28, no. 3, pp. 237-250, 2001.

[11] M. E. Kraenzlin and C. Meier, "Parathyroid hormone analogues in the treatment of osteoporosis," Nature Reviews Endocrinology, vol. 7, no. 11, pp. 647-656, 2011.

[12] P. Chen, C. P. Jerome, D. B. Burr et al., "Interrelationships between bone microarchitecture and strength in ovariectomized monkeys treated with teriparatide," Journal of Bone and Mineral Research, vol. 22, no. 6, pp. 841-848, 2007.

[13] R. M. Neer, C. D. Arnaud, J. R. Zanchetta et al., "Effect of parathyroid hormone (1-34) on fractures and bone mineral density in postmenopausal women with osteoporosis," The New England Journal of Medicine, vol. 344, no. 19, pp. 1434-1441, 2001.

[14] J.-M. Kaufman, E. Orwoll, S. Goemaere et al., “Teriparatide effects on vertebral fractures and bone mineral density in men with osteoporosis: treatment and discontinuation of therapy," Osteoporosis International, vol. 16, no. 5, pp. 510-516, 2005.

[15] R. Lindsay, W. H. Scheele, R. Neer et al., "Sustained vertebral fracture risk reduction after withdrawal of teriparatide in postmenopausal women with osteoporosis," Archives of Internal Medicine, vol. 164, no. 18, pp. 2024-2030, 2004.

[16] E. S. Orwoll, W. H. Scheele, S. Paul et al., "The effect of teriparatide [human parathyroid hormone (1-34)] therapy on bone density in men with osteoporosis," Journal of Bone and Mineral Research, vol. 18, no. 1, pp. 9-17, 2003.

[17] T. J. Martin, J. M. W. Quinn, M. T. Gillespie, K. W. Ng, M. A. Karsdal, and N. A. Sims, "Mechanisms involved in skeletal anabolic therapies," Annals of the New York Academy of Sciences, vol. 1068, no. 1, pp. 458-470, 2006.
[18] M. R. Rubin and J. P. Bilezikian, "The anabolic effects of parathyroid hormone therapy," Clinics in Geriatric Medicine, vol. 19, no. 2, pp. 415-432, 2003.

[19] M. Ito, R. Oishi, M. Fukunaga et al., "The effects of once-weekly teriparatide on hip structure and biomechanical properties assessed by CT," Osteoporosis International, vol. 25, no. 3, pp. 1163-1172, 2014.

[20] S. Komatsubara, S. Mori, T. Mashiba et al., "Human parathyroid hormone (1-34) accelerates the fracture healing process of woven to lamellar bone replacement and new cortical shell formation in rat femora," Bone, vol. 36, no. 4, pp. 678-687, 2005.

[21] M. Komrakova, E. K. Stuermer, C. Werner et al., "Effect of human parathyroid hormone hPTH (1-34) applied at different regimes on fracture healing and muscle in ovariectomized and healthy rats," Bone, vol. 47, no. 3, pp. 480-492, 2010.

[22] M. J. Gardner, M. C. H. Van Der Meulen, J. Carson et al., "Role of parathyroid hormone in the mechanosensitivity of fracture healing," Journal of Orthopaedic Research, vol. 25, no. 11, pp. 1474-1480, 2007.

[23] Y. F. Li, C. C. Zhou, J. H. Li et al., "The effects of combined human parathyroid hormone (1-34) and zoledronic acid treatment on fracture healing in osteoporotic rats," Osteoporosis International, vol. 23, no. 4, pp. 1463-1474, 2012.

[24] T.-W. Huang, T.-Y. Yang, K.-C. Huang, K.-T. Peng, M. S. Lee, and R. W.-W. Hsu, "Effect of teriparatide on unstable pertrochanteric fractures," BioMed Research International, vol. 2015, Article ID 568390, 8 pages, 2015.

[25] A. M. Cheung, L. Tile, N. Austin et al., "Use of teriparatide in patients with atypical femur fractures," Journal of Bone and Mineral Research, vol. 26, 2011.

[26] A. M. Cheung, J. Adachi, A. Khan et al., "Effect of teriparatide on healing of atypical femoral fractures: a systemic review," Journal of Bone and Mineral, vol. 22, no. 4, pp. 183-189, 2015.

[27] N. K. Kanakaris, R. M. West, and P. V. Giannoudis, "Enhancement of hip fracture healing in the elderly: evidence deriving from a pilot randomized trial," Injury, vol. 46, no. 8, pp. 1425$1428,2015$.

[28] T. Johansson, "PTH 1-34 (teriparatide) may not improve healing in proximal humerus fractures," Acta Orthopaedica, vol. 87, no. 1, pp. 79-82, 2016.

[29] J. Goldhahn, J.-M. Féron, J. Kanis et al., "Implications for fracture healing of current and new osteoporosis treatments: an ESCEO consensus paper," Calcified Tissue International, vol. 90, no. 5, pp. 343-353, 2012.

[30] P. Peichl, L. A. Holzer, R. Maier, and G. Holzer, "Parathyroid hormone 1-84 accelerates fracture-healing in pubic bones of elderly osteoporotic women," Journal of Bone and Joint Surgery-Series A, vol. 93, no. 17, pp. 1583-1587, 2011.

[31] P. Aspenberg, H. K. Genant, T. Johansson et al., "Teriparatide for acceleration of fracture repair in humans: a prospective, randomized, double-blind study of 102 postmenopausal women with distal radial fractures," Journal of Bone and Mineral Research, vol. 25, no. 2, pp. 404-414, 2010.

[32] A. D. Oxman, "Grading quality of evidence and strength of recommendations," British Medical Journal, vol. 328, no. 7454, pp. 1490-1494, 2004.

[33] J. P. T. Higgins, S. G. Thompson, J. J. Deeks, and D. G. Altman, "Measuring inconsistency in meta-analyses," British Medical Journal, vol. 327, no. 7414, pp. 557-560, 2003.

[34] R. DerSimonian and N. Laird, "Meta-analysis in clinical trials," Controlled Clinical Trials, vol. 7, no. 3, pp. 177-188, 1986. 
[35] H. K. Song, S. J. Kim, J. H. Lee, and K. H. Yang, "Intermittent parathyroid hormone treatment for stimulation of callus formation in elderly patients," Journal of the Korean Fracture Society, vol. 25, no. 4, pp. 295-299, 2012.

[36] P. Aspenberg and T. Johansson, "Teriparatide improves early callus formation in distal radial fractures," Acta Orthopaedica, vol. 81, no. 2, pp. 234-236, 2010.

[37] T. Nakazawa, A. Nakajima, K. Shiomi, H. Moriya, T. A. Einhorn, and M. Yamazaki, "Effects of low-dose, intermittent treatment with recombinant human parathyroid hormone (134) on chondrogenesis in a model of experimental fracture healing," Bone, vol. 37, no. 5, pp. 711-719, 2005.

[38] A. Nakajima, N. Shimoji, K. Shiomi et al., "Mechanisms for the enhancement of fracture healing in rats treated with intermittent low-dose human parathyroid hormone (1-34)," Journal of Bone and Mineral Research, vol. 17, no. 11, pp. 2038-2047, 2002.

[39] S. Kakar, T. A. Einhorn, S. Vora et al., "Enhanced chondrogenesis and Wnt signaling in PTH-treated fractures," Journal of Bone and Mineral Research, vol. 22, no. 12, pp. 1903-1912, 2007.

[40] J. D. Bashutski, R. M. Eber, J. S. Kinney et al., "Teriparatide and osseous regeneration in the oral cavity," The New England Journal of Medicine, vol. 363, no. 25, pp. 2396-2405, 2010.

[41] N. Kobayashi, Y. Inaba, M. Uchiyama, H. Ike, S. Kubota, and T. Saito, "Teriparatide versus alendronate for the preservation of bone mineral density after total hip arthroplasty-a randomized controlled trial," Journal of Arthroplasty, vol. 31, no. 1, pp. 333-338, 2016.

[42] T. Matsumoto, M. Ando, and S. Sasaki, "Effective treatment of delayed union of a lumbar vertebral fracture with daily administration of teriparatide in a patient with diffuse idiopathic skeletal hyperostosis," European Spine Journal, vol. 24, supplement 4, pp. S573-S576, 2015.

[43] Y.-K. Lee, Y.-C. Ha, and K.-H. Koo, “Teriparatide, a nonsurgical solution for femoral nonunion? A report of three cases," Osteoporosis International, vol. 23, no. 12, pp. 2897-2900, 2012.

[44] S. Chintamaneni, K. Finzel, and B. L. Gruber, "Successful treatment of sternal fracture nonunion with teriparatide," Osteoporosis International, vol. 21, no. 6, pp. 1059-1063, 2010.

[45] K. Tamai, K. Takamatsu, and K. Kazuki, "Successful treatment of nonunion with teriparatide after failed ankle arthrodesis for Charcot arthropathy," Osteoporosis International, vol. 24, no. 10, pp. 2729-2732, 2013.

[46] O. Johnell and J. A. Kanis, "An estimate of the worldwide prevalence and disability associated with osteoporotic fractures," Osteoporosis International, vol. 17, no. 12, pp. 1726-1733, 2006.

[47] S. P. Hozo, B. Djulbegovic, and I. Hozo, "Estimating the mean and variance from the median, range, and the size of a sample," BMC Medical Research Methodology, vol. 5, article 13, 2005. 


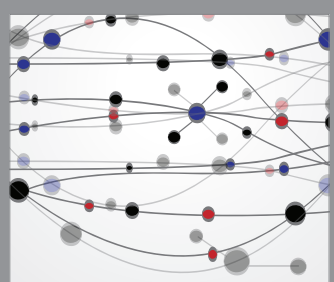

The Scientific World Journal
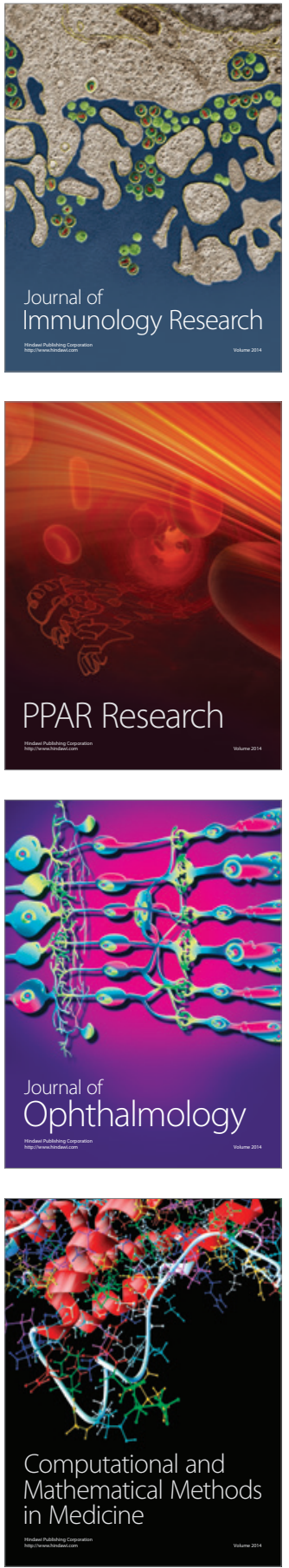

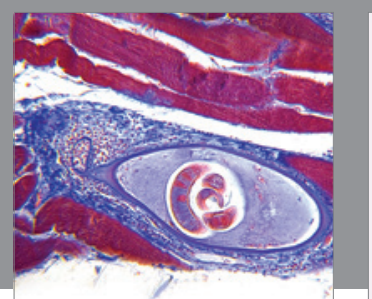

Gastroenterology Research and Practice

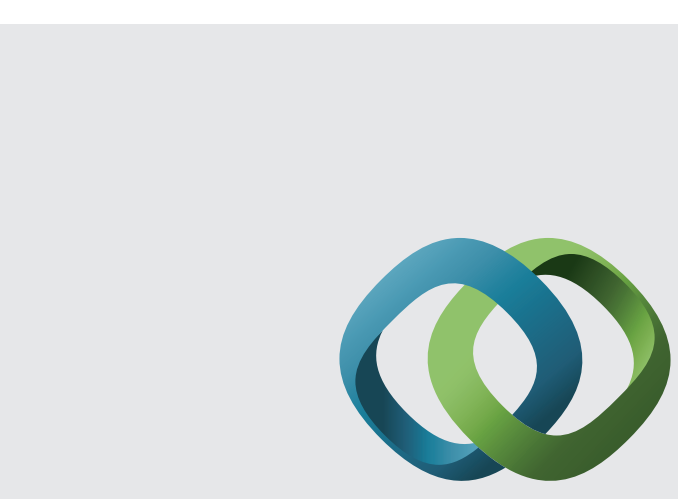

\section{Hindawi}

Submit your manuscripts at

http://www.hindawi.com
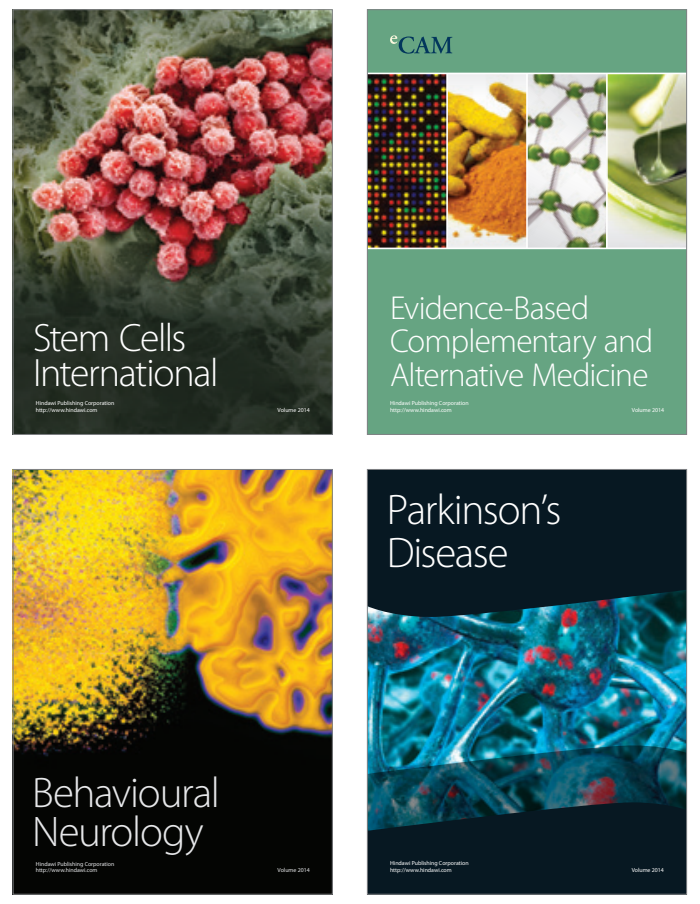
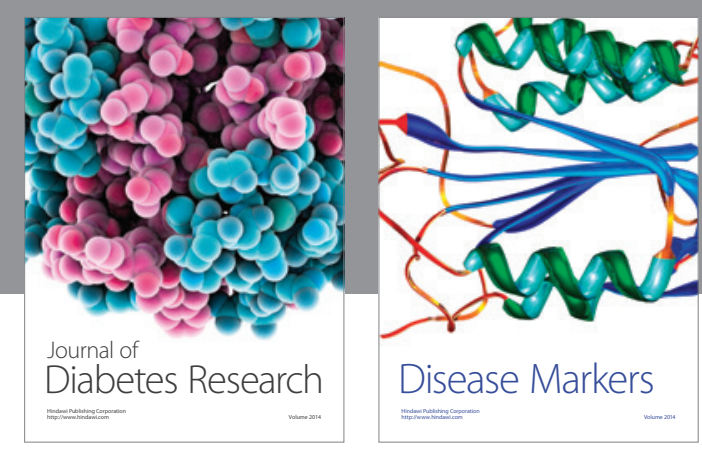

Disease Markers
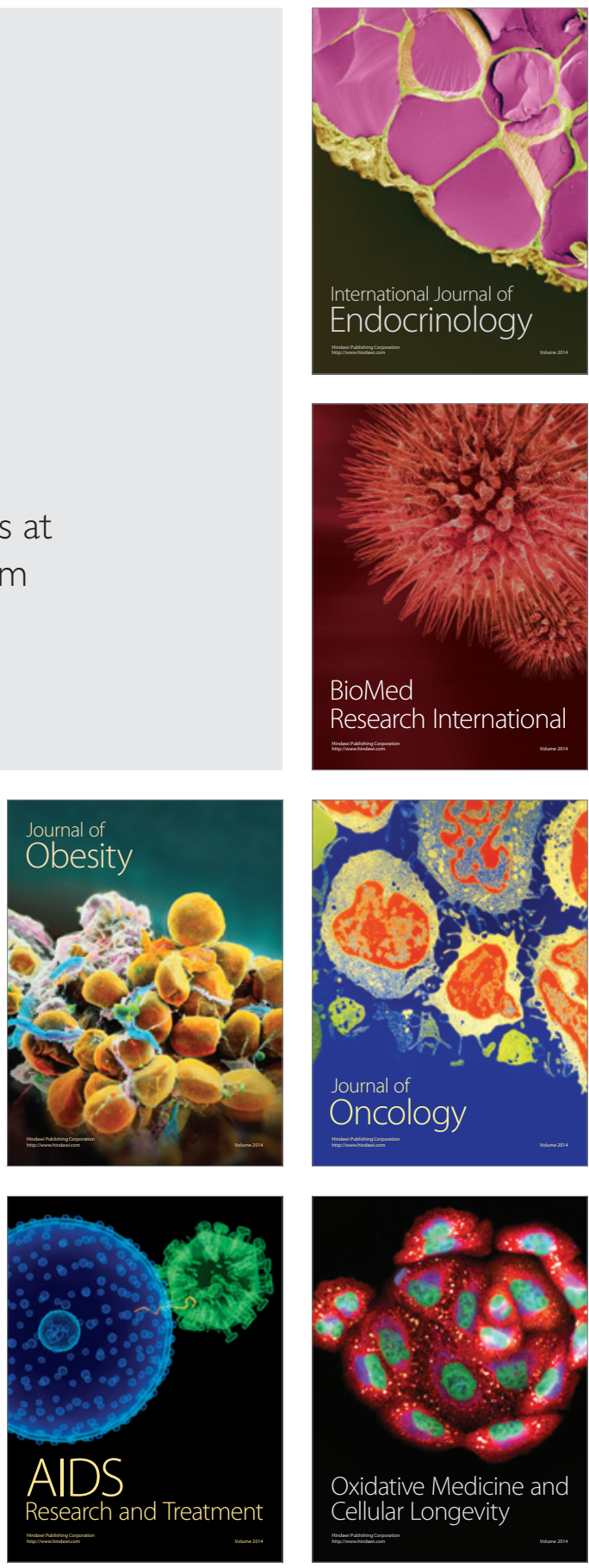